\title{
Photonic multipartite entanglement conversion using nonlocal operations
}

\author{
T. Tashima,,${ }^{1,2,3,4}$ M. S. Tame, ${ }^{4,5}$ Ş. K. Özdemir, ${ }^{6}$ F. Nori, ${ }^{7,8}$ M. Koashi, ${ }^{9}$ and H. Weinfurter ${ }^{1,2}$ \\ ${ }^{1}$ Department für Physik, Ludwig-Maximilians-Universität, D-80799 München, Germany \\ ${ }^{2}$ Max-Planck-Institut für Quantenoptik, Hans-Kopfermann-Strasse 1, D-85748 Garching, Germany \\ ${ }^{3}$ Division of Materials Physics, Department of Materials Engineering Science, \\ Graduate School of Engineering Science, Osaka University, Toyonaka, Osaka 560-8531, Japan \\ ${ }^{4}$ School of Chemistry and Physics, University of KwaZulu-Natal, Durban 4001, South Africd * \\ ${ }^{5}$ National Institute for Theoretical Physics, KwaZulu-Natal, South Africd \\ ${ }^{6}$ Department of Electrical and Systems Engineering, \\ Washington University in St. Louis, St. Louis, MO 63130 USA 甲 $^{7}$ \\ ${ }^{7}$ Center for Emergent Matter Science, RIKEN, Saitama 351-0198, Japan \\ ${ }^{8}$ Physics Department, University of Michigan, Ann Arbor, Michigan 48109-1040, USA \\ ${ }^{9}$ Photon Science Center, The University of Tokyo, Bunkyo-ku, 113-8656, Japan
}

(Dated: September 11, 2018)

\begin{abstract}
We propose a simple setup for the conversion of multipartite entangled states in a quantum network with restricted access. The scheme uses nonlocal operations to enable the preparation of states that are inequivalent under local operations and classical communication, but most importantly does not require full access to the states. It is based on a flexible linear optical conversion gate that uses photons, which are ideally suited for distributed quantum computation and quantum communication in extended networks. In order to show the basic working principles of the gate, we focus on converting a four-qubit entangled cluster state to other locally inequivalent four-qubit states, such as the GHZ and symmetric Dicke state. We also show how the gate can be incorporated into extended graph state networks, and can be used to generate variable entanglement and quantum correlations without entanglement but nonvanishing quantum discord.
\end{abstract}

PACS numbers: 03.67.Mn, 03.67.-a, 42.50.-p

\section{INTRODUCTION}

Entanglement between two or more particles is an elementary resource for a variety of quantum communication and computing tasks [1-5]. Recently, sophisticated quantum network architectures relying on resources with different entanglement structures among the nodes of a network have been proposed for distributed quantum communication and computing [6 - 8 ]. In order to make full use of the nodes of a network, it is important to identify and to prepare the optimal shared resource for a given task, as well as to understand the equivalence relations among different entangled resource states and the inequivalent classes of entanglement.

Progress in the general study of multipartite entanglement was stimulated by the finding that entangled states of three qubits cannot be converted into each other by local operations and classical communication (LOCC) [10, 16]. While for bipartite pure states there is only one class of states under LOCC, the situation changes dramatically for more qubits. For example, for four parties, Greenberger-Horne-Zeilinger (GHZ) states 9], W states [10-12], Cluster states [3, 4] and Dicke states 13] come from inequivalent entanglement classes [14, 15].

\footnotetext{
*Electronic address: ttoshi@oregano.ocn.ne.jp

†Electronic address: markstame@gmail.com

‡Electronic address: ozdemir@wustl.edu
}

a)

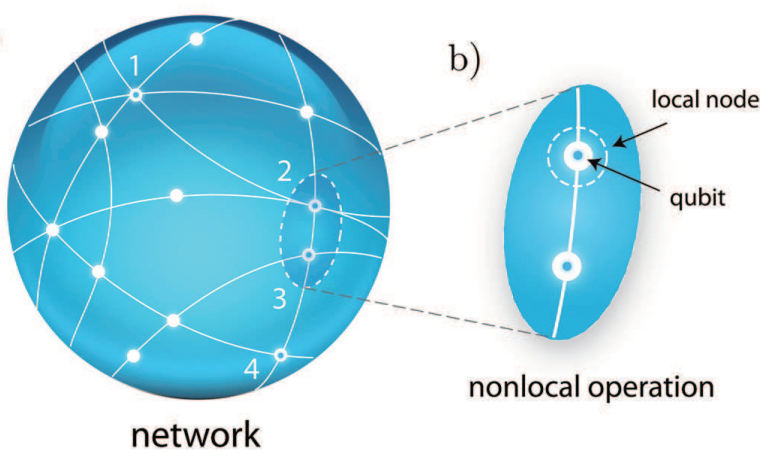

FIG. 1: Quantum network with restricted access. We consider a nonlocal conversion gate that operates on qubits from two local nodes of the network, as shown in panel (b), and enables the conversion of different types of multipartite entangled states for quantum networking applications. While we focus on four-qubit entangled states in our study, the overall principle could be extended to larger more complex networks due to the operating range of the conversion gate.

This classification led to a series of efforts to find methods and schemes to prepare and characterize these states in different physical setups [17], ranging from photonics 5] and nuclear magnetic resonance 18], to ion traps [19, 20] and superconducting circuits [21, 22]. Studies have also focused on forming complete toolboxes of physically realizable operations and gates to manipulate, expand and fuse entangled states of a certain type to form larger states of the same type such that entangled 
networks can be formed $23,35,39,46]$. In parallel to this work, there has also been much interest in the use of entangled states in quantum information processing tasks, especially in finding tasks for which the states from one entanglement class may be more efficient than one from another class. For example, cluster states and graph states are universal resources for quantum computing [3, 4], GHZ states have been proposed as resources for achieving consensus in distributed networks without classical post-processing [47] and $\mathrm{W}$ states have been proposed as resources for leader election in anonymous quantum networks [47] and asymmetric telecloning [48]. From a network perspective, the qubits are distributed to the nodes where the users have access only to a limited number of qubits. Typically they make use of classical communication channels to perform individually or collectively an assigned task [47-49]. In some cases, two nodes of a network may be close enough to each other such that joint operations can be carried out with a small overhead in communication, as shown in Fig. 1, Such a scenario could also describe the case where a node of a network is also the node of another network and holds two or more qubits belonging to different networks. This 'nonlocal' node could manipulate qubits from different networks for the purpose of fusing those networks into a larger merged network. This and similar tasks are of considerable importance as they help us to understand how to efficiently exploit multipartite entanglement for quantum communication protocols and how to design practical applications.

Recent work has focused on the transformation of different types of entangled states into each other by local one and two-qubit operations while allowing classical communication among all the nodes. For example, Kiesel et al. [50] demonstrated that a four-partite symmetric Dicke state can be used to prepare a W state among three parties if one of the parties in the network projects their qubit onto the computational basis. The initial symmetric Dicke state can be used in certain quantum versions of classical games whereas the final $\mathrm{W}$ state cannot be used [51, 52]. On the other hand, the final W state can be used for asymmetric telecloning of a qubit [48]. Along the same lines, another interesting work is that of Walther et al. [53], who showed that by the application of appropriate generalized measurements on a tripartite GHZ state, one can obtain a state whose fidelity to a tripartite $\mathrm{W}$ state approaches one, when the probability of success approaches zero. Tashima et al. [31], on the other hand, have proposed a series of optical gates that can prepare $\mathrm{W}$ states of arbitrary size by accessing only one qubit of the $\mathrm{W}$ state. They also introduced a scheme in which two parties sharing a pair of Bell states can prepare tripartite $\mathrm{W}$ states and expand them to larger sizes with the help of ancillary qubits, again by accessing only one qubit in the network [31, 32, 35].

In this work we go beyond those concepts by introducing a scheme that uses 'bilocal' operations to enable the preparation of resource states in a network that are inequivalent under LOCC, but crucially does not require

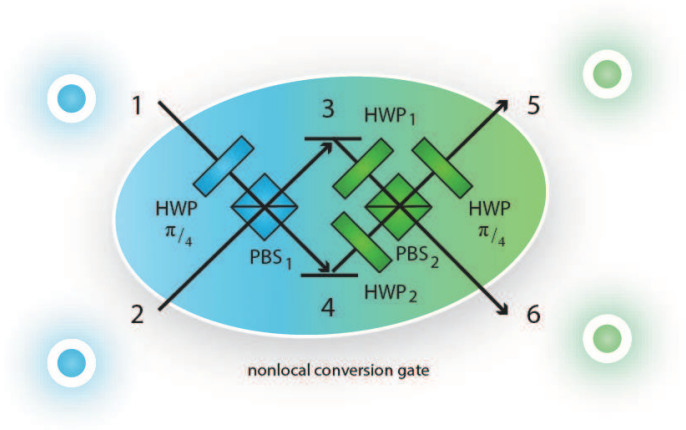

FIG. 2: Nonlocal gate using linear optics. The gate is used in our restricted-access network scenario to convert between different types of entanglement structures.

full access to all of the resource state's constituent elements. We call the operations bilocal, as they involve a nonlocal two-qubit gate and local one-qubit operations. Here, the two qubits operated on by the nonlocal gate need to be in close proximity, whereas the other qubits do not. This is in contrast to a fully nonlocal operation that would require all qubits to be in close proximity for it to be applied. Our scheme employs a flexible linear optical conversion gate. The gate can be used to generate variable entanglement, as well as quantum correlations without entanglement captured by the quantum discord. While we focus on four-qubit entangled states in our study, e.g. cluster, GHZ and Dicke states, the overall principle can be extended to larger and more complex networks due to the flexible operation of the gate. We highlight this extension briefly in our work.

The paper is organized as follows: In Sec. II, we describe the principles of the nonlocal gate used in our conversion scheme and highlight its overall range of operation. We also show how it can prepare states that are either entangled or have nonzero discord. In Sec. III, we show that the gate can be used to convert a four-qubit cluster state into other four-qubit entangled states by operating nonlocally on only two qubits. In Sec. IV we show some examples of incorporating the nonlocal gate in an extended graph state network. Finally, in Sec. V we provide a brief summary and outlook.

\section{NONLOCAL GATE FOR PHOTONIC STATE CONVERSION}

Let us start by introducing the nonlocal two-qubit gate for photonic state conversion. The gate shown in Fig. 2 is composed of two polarizing beamsplitters (PBSs) and four half-wave plates (HWPs). At the most basic level it functions as a tunable polarization-dependent beamsplitter (PDBS). However, its advantage over the standard PDBS already used in experiments [32, 36, 37] is that as the operation of the individual components can be adjusted easily the configuration is far more flexible 
and readily implemented experimentally. This is in direct contrast to a bulk $\mathrm{PDBS}$, whose operation is set once fabrication has been completed and cannot be changed. The flexibility of this tunable PDBS complements well the work on a tunable polarization-independent beamsplitter [38]. In this section we consider the individual components of the gate in order to derive the necessary expressions to demonstrate its working principles and range of operation. We then discuss how it can be used to generate entanglement and more general quantum correlations.

\section{A. Working principle of the conversion gate}

The conversion gate is shown in Fig. 2, Its successful operation is based on postselection such that one photon is detected in each of the output modes, labeled 5 and 6 . The Kraus operator $E_{0}$ of the gate transforms the input state $\rho_{i n}=\left|\psi_{i n}\right\rangle\left\langle\psi_{i n}\right|$ according to

$$
\rho_{i n} \rightarrow \frac{E_{0}\left|\psi_{i n}\right\rangle\left\langle\psi_{i n}\right| E_{0}^{\dagger}}{p_{s}}
$$

where $p_{s}=\operatorname{Tr}\left(E_{0}\left|\psi_{i n}\right\rangle\left\langle\psi_{i n}\right| E_{0}^{\dagger}\right)$ is the success probability. Here, the action of the HWPs in modes 3 and 4 for the polarization degree of freedom of one photon is given by

$$
\begin{aligned}
|H\rangle_{j} & \rightarrow \cos \left(2 \theta_{l}\right)|H\rangle_{j}+\sin \left(2 \theta_{l}\right)|V\rangle_{j} \\
|V\rangle_{j} & \rightarrow \sin \left(2 \theta_{l}\right)|H\rangle_{j}-\cos \left(2 \theta_{l}\right)|V\rangle_{j} .
\end{aligned}
$$

where $j=3,4$ labels the modes of the HWPs and $l=1,2$ labels the HWPs. When the input state is $|H V\rangle_{j}$, the action of the HWPs in modes 3 and 4 is given by

$$
\begin{aligned}
|H V\rangle_{j} \rightarrow & \sqrt{2} \cos \left(2 \theta_{l}\right) \sin \left(2 \theta_{l}\right)|2 H\rangle_{j} \\
& -\left(\cos ^{2}\left(2 \theta_{l}\right)-\sin ^{2}\left(2 \theta_{l}\right)\right)|H V\rangle_{j} \\
& +\sqrt{2} \sin \left(2 \theta_{l}\right) \cos \left(2 \theta_{l}\right)|2 V\rangle_{j},
\end{aligned}
$$

where we have used the definition $|H V\rangle_{j}$ for one horizontal polarized photon and one vertical polarized photon in the same mode $j$, and $|2 H\rangle_{j}\left(|2 V\rangle_{j}\right)$ for two horizontal (vertical) polarized photons in mode $j$. In addition, the first PBS applies the unitary transformations: $|H\rangle_{1} \rightarrow|H\rangle_{4},|H\rangle_{2} \rightarrow|H\rangle_{3},|V\rangle_{1} \rightarrow|V\rangle_{3}$ and $|V\rangle_{2} \rightarrow|V\rangle_{4}$, and the second PBS applies similar transformations: $|H\rangle_{3} \rightarrow|H\rangle_{6},|H\rangle_{4} \rightarrow|H\rangle_{5},|V\rangle_{3} \rightarrow|V\rangle_{5}$ and $|V\rangle_{4} \rightarrow|V\rangle_{6}$. By combining all the unitary operations of the HWPs and PBSs, the Kraus operator of the nonlocal gate can be written as

$$
\begin{aligned}
E_{0}= & \left(\alpha_{1}-\beta_{1}\right)|H H\rangle_{5612}\langle H H| \\
& +\left(\alpha_{2}-\beta_{2}\right)|V V\rangle_{5612}\langle V V| \\
& +\mu_{1}|H V\rangle_{56}{ }_{12}\left\langle H V\left|-\mu_{2}\right| V H\right\rangle_{56}{ }_{12}\langle H V| \\
& +\mu_{1}|V H\rangle_{56}{ }_{12}\left\langle V H\left|-\mu_{2}\right| H V\right\rangle_{56}{ }_{12}\langle V H|
\end{aligned}
$$

where $\alpha_{l}=\cos ^{2}\left(2 \theta_{l}\right), \quad \beta_{l}=\sin ^{2}\left(2 \theta_{l}\right), \quad \mu_{1}=$ $\cos \left(2 \theta_{1}\right) \cos \left(2 \theta_{2}\right)$ and $\mu_{2}=\sin \left(2 \theta_{1}\right) \sin \left(2 \theta_{2}\right)$. The Kraus
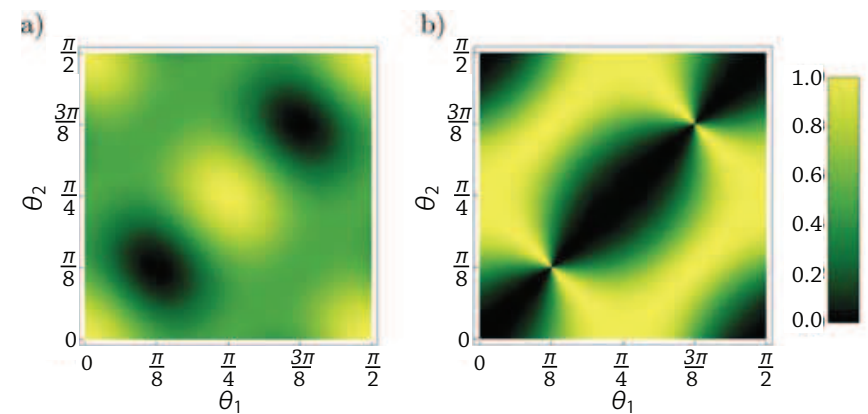

FIG. 3: (a): Success probability for the input state $\left|\psi_{i n}\right\rangle=$ $|+\rangle|+\rangle$ for the conversion gate operating with HWPs as polarization rotators at angles $\theta_{1}$ and $\theta_{2}$. (b): Entanglement generated from this input as quantified by the concurrence.

operator $E_{0}$ corresponds to a successful gate operation with probability $p_{s}$, where each of the output ports (modes 5 and 6 ) has one photon. It is clear that by tuning the rotation angles of $\mathrm{HWP}_{1}$ and $\mathrm{HWP}_{2}$, the gate acts as a tunable PDBS, enabling the construction of different Kraus operators. This makes it possible to perform different tasks using this simple linear optical construction.

\section{B. Entanglement generation}

We now show some basic examples that demonstrate the entangling power of the conversion gate and its PDBS ability. In Fig. 3, we show the success probability and a range of states with different amounts of entanglement generated by tuning the angles $\theta_{1}$ and $\theta_{2}$, as quantified by the concurrence [54]. Here, the input state is $\left|\psi_{i n}\right\rangle=$ $|+\rangle|+\rangle$, where $|+\rangle=(|H\rangle+|V\rangle) / \sqrt{2}$. Applying the Kraus operator $E_{0}$ of Eq. (44) on this input state yields

$$
\begin{aligned}
E_{0}\left|\psi_{i n}\right\rangle= & \frac{1}{2}\left[\left(\alpha_{1}-\beta_{1}\right)|H H\rangle+\left(\alpha_{2}-\beta_{2}\right)|V V\rangle\right. \\
& \left.+\sqrt{2}\left(\mu_{1}-\mu_{2}\right)\left|\Psi^{+}\right\rangle\right]
\end{aligned}
$$

where $\left|\Psi^{+}\right\rangle=(|H V\rangle+|V H\rangle) / \sqrt{2}$.

First, in order to prepare the Bell state $\left|\Psi^{+}\right\rangle$from the state space of the output in Eq. (5) we need to set $\alpha_{1}-\beta_{1}=0$ and $\alpha_{2}-\beta_{2}=0$ by rotating the angles of the HWPs in modes 3 and 4 as $\theta_{1}=(2 k+1) \frac{\pi}{8}$ and $\theta_{2}=(2 n+1) \frac{\pi}{8}$, respectively, for $k$ and $n$ taking nonnegative integer values. The success probability is maximized when $\mu_{1}-\mu_{2}=\cos 2\left(\theta_{1}+\theta_{2}\right)=\mp 1$, leading to $\theta_{1}+\theta_{2}=m \pi / 2$ for some integer $m$. These three equations are satisfied simultaneously when $(2 k+1) \frac{\pi}{8}+(2 n+$ 1) $\frac{\pi}{8}=m \frac{\pi}{2}$. This can be reformulated to $k+n+1=2 m$, implying that $k+n$ should be an odd number. In other words, when $k$ is even $n$ is odd and vice versa. Thus the solution we are looking for is $\mu_{1}=-\mu_{2}= \pm 1 / 2$ resulting in $E_{0}= \pm\left|\Psi^{+}\right\rangle\left\langle\Psi^{+}\right|$. Consequently, the Kraus operator $E_{0}$ prepares the output state $\rho_{\text {out }}=\left|\Psi^{+}\right\rangle\left\langle\Psi^{+}\right|$with the success probability $p_{s}=1 / 2$. In order to give an 
idea of the robustness of the success probability to variations in the optical components, as in an experimental implementation, we performed a Monte Carlo simulation, varying the angles $\theta_{1}$ and $\theta_{2}$ of the wave plates around their ideal values. We set a range of variation for the angles of $\pm 10 \%$ from the ideal value. Performing 5000 runs (to reach asymptotic behaviour) we find an average success probability of $p_{s}=0.509 \pm 0.010$, which clearly shows that the success probability is quite robust to realistic perturbations.

Similarly, we see from Eq. (5) that if we were able to set $\mu_{1}-\mu_{2}=0$ and $\alpha_{1}-\beta_{1}= \pm\left(\alpha_{2}-\beta_{2}\right)$ this would prepare the Bell state $\left|\Phi^{ \pm}\right\rangle=[|H H\rangle \pm|V V\rangle] / \sqrt{2}$. For the former equality, we find $\theta_{1}+\theta_{2}=(2 m+1) \frac{\pi}{4}$ for non-negative integer numbers $m$. The latter equality is satisfied when $4 \theta_{2}=4 \theta_{1}+2 n \pi$ and $4 \theta_{2}=4 \theta_{1}+(2 k+1) \pi$, respectively for + and - signs. It is easy to show under these conditions that the case with the $+\operatorname{sign}$ gives $\alpha_{1}-\beta_{1}=\alpha_{2}-\beta_{2}=0$ which is not desirable. On the other hand, the case with the $-\operatorname{sign}$ leads to $\theta_{1}=(m-k) \frac{\pi}{4}$ and $\theta_{2}=(m+k+1) \frac{\pi}{4}$ for which $\alpha_{1}-\beta_{1}=-\left(\alpha_{2}-\beta_{2}\right)$ is always satisfied. The above implies that we can prepare only the state $\left|\Phi^{-}\right\rangle$ with the success probability $p_{s}=1 / 2$. In this case, we obtain $\alpha_{1}=\beta_{2}=\mu_{1}=\mu_{2}=0$ and $\alpha_{2}=\beta_{1}=1$, leading to $E_{0}=|H H\rangle\langle H H|-| V V\rangle\langle V V|$ and the output state $\rho_{\text {out }}=\left|\Phi^{-}\right\rangle\left\langle\Phi^{-}\right|$with success probability $p_{s}=$ $1 / 2$. A Monte Carlo simulation with a $\pm 10 \%$ variation on the wave plate angles gives a success probability of $p_{s}=0.481 \pm 0.021$.

It is clear that starting with the input state $\left|\psi_{\text {in }}\right\rangle=$ $|+\rangle|+\rangle$ (or $\left|\psi_{\text {in }}\right\rangle=|-\rangle|-\rangle$, where $|-\rangle=(|H\rangle-$ $|V\rangle) / \sqrt{2})$ ), the proposed gate can prepare a range of states with various amounts of entanglement as seen in Fig. 3, including separable and maximally entangled states, by simply setting the rotation angles of $\mathrm{HWP}_{1}$ and $\mathrm{HWP}_{2}$ correctly. It is also noted that the output state resulting from the input state $\left|\psi_{\text {in }}\right\rangle=|+\rangle|-\rangle$ or $\left|\psi_{i n}\right\rangle=|-\rangle|+\rangle$ can be obtained from the input state $\left|\psi_{\text {in }}\right\rangle=|+\rangle|+\rangle$ by locally compensating the phase shift in either mode 5 or 6 .

\section{Discord generation}

We now give a brief example of how the conversion gate can be used to generate quantum correlations different from entanglement. Here, the quantum discord [55 57] allows the quantification of nonclassical correlations that exist between quantum systems even when the entanglement is zero. Recently it has been shown that discord can be used as a resource for various types of quantum protocols that are useful in a quantum network scenario, including remote state preparation [58], encoding information that only coherent interactions can extract [59] and verification that untrusted parties can implement entanglement operations [60]. Discord represents the quantum component of correlations between two systems, $A$ and $B$, and is defined as $\delta(A \mid B)=I(A, B)-J(A \mid B)$ with a)

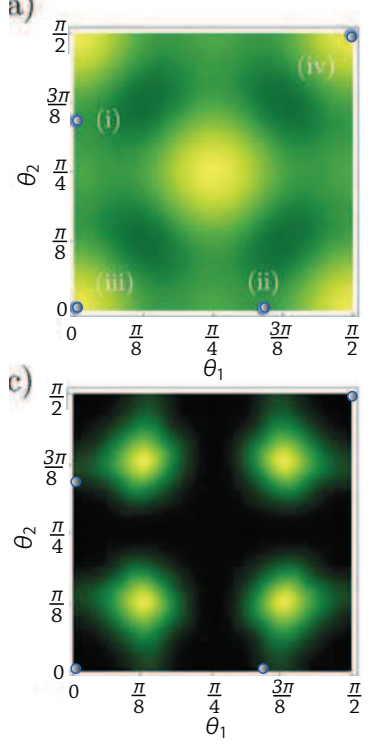

b)
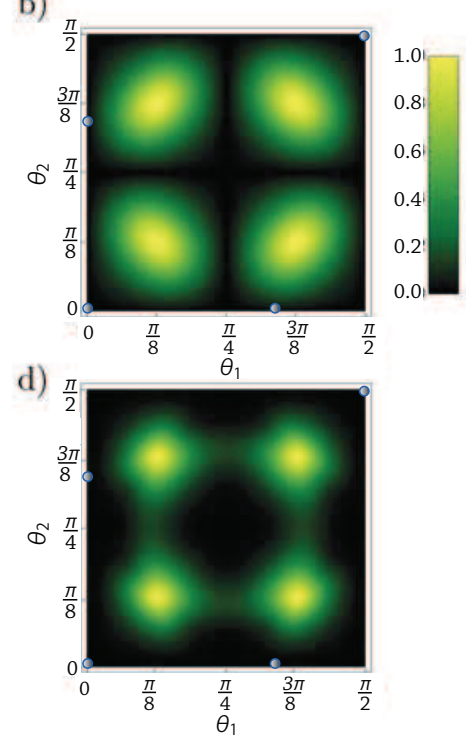

FIG. 4: Generation of nonclassical correlations by the conversion gate that are not due to entanglement. (a): Success probability of the conversion gate. Here points (i) and (ii) correspond to the output state $\frac{5}{7}|H\rangle\langle H|\otimes| \phi\rangle\left\langle\phi\left|+\frac{2}{7}\right| V\right\rangle\langle V| \otimes$ $|+\rangle\langle+|$, where $|\phi\rangle=(2|H\rangle-|V\rangle) / \sqrt{5}$. Points (iii) and (iv) correspond to the output state $\frac{1}{2} \mathbb{1} \otimes|+\rangle\langle+|$. (b): Entanglement generated as quantified by the concurrence. (c): Discord generated with respect to measurements performed on the second qubit. (d): Discord generated with respect to measurements performed on the first qubit.

$I(A, B)$ and $J(A \mid B)$ given by

$$
\begin{aligned}
& I(A, B)=S\left(\rho_{A}\right)+S\left(\rho_{B}\right)-S\left(\rho_{A B}\right) \\
& J(A \mid B)=S\left(\rho_{A}\right)-\min _{\left\{\Pi_{b}\right\}} \sum p_{b} S\left(\rho_{A \mid b}\right)
\end{aligned}
$$

where $S(\rho)$ is the von Neumann entropy $S(\rho)=$ $-\operatorname{Tr} \rho \log \rho, \rho_{A}=\operatorname{Tr}_{B} \rho_{A B}, \rho_{B}=\operatorname{Tr}_{A} \rho_{A B},\left\{\Pi_{b}\right\}$ is a positive operator valued measure on system $B$, and $p_{b}=$ $\operatorname{Tr}\left[\rho \Pi_{b}\right]$ is the probability of obtaining measurement outcome $b$ that leaves $A$ in the conditional state $\rho_{A \mid b}$. In general the discord is not symmetric, $\delta(A \mid B) \neq \delta(B \mid A)$, but if it is non-zero with respect to measurements on at least one system, then nonclassical correlations exist. In addition, when the entanglement is zero and the discord is non-zero with respect to measurements on one or both of systems $A$ and $B(\delta(B \mid A) \neq 0$ or $\delta(A \mid B) \neq 0)$, then there are quantum correlations present that are not due to entanglement. In order to show that the conversion gate generates such quantum correlations from initial product states without entanglement we input the state $\rho_{\text {in }}=\frac{1}{2} \mathbb{1} \otimes|+\rangle\langle+|$. In Fig. 4 (a) the success probability is shown as the angles $\theta_{1}$ and $\theta_{2}$ of the gate are modified. In Fig. 4 (b) the corresponding entanglement generated by the gate is shown, as quantified by the concurrence.

In Figs. 4 (c) and $4(\mathrm{~d})$, we give the discords $\delta(A \mid B)$ and $\delta(B \mid A)$ generated in the output state with respect to 
measurements performed on the second and first qubit in the conversion gate, where $A$ corresponds to the first qubit in output mode 5 and $B$ corresponds to the second qubit in output mode 6 . When $\theta_{1}=0$ and $\theta_{2}=\pi / 3$ (point (i)) or $\theta_{1}=\pi / 3$ and $\theta_{2}=0$ (point (ii)) the output state from the gate has no entanglement in Fig. 4 (b), but interestingly has non-zero discord (See in Fig. 4 (c)). The output state for these points is $\frac{5}{7}|H\rangle\langle H|\otimes| \phi\rangle\langle\phi|+$ $\frac{2}{7}|V\rangle\langle V|\otimes|+\rangle\langle+|$, where $|\phi\rangle=(2|H\rangle-|V\rangle) / \sqrt{5}$ and has a discord of $\delta(A \mid B) \simeq 0.082$. The success probability is 0.438 and a Monte Carlo simulation with a $\pm 10 \%$ variation on the wave plate angles gives $p_{s}=0.446 \pm 0.023$.

This way the conversion gate can also be used to generate states with quantum correlations that are not due to entanglement and may be used for various quantum tasks in a network scenario [58 60], most notably as resources in quantum cryptography [61].

\section{STATE CONVERSION}

One of the powerful features of the nonlocal gate is the ability to convert quantum states belonging to one type of state into another that is not LOCC equivalent.

Starting with a four-qubit linear cluster state given by

$$
\begin{aligned}
\left|\mathrm{C}_{4}\right\rangle= & \frac{1}{2}(|H H H H\rangle+|H H V V\rangle \\
& +|V V H H\rangle-|V V V V\rangle),
\end{aligned}
$$

we will show how the action of the conversion gate shown in Fig. 2 on two qubits of the state $\rho_{i n}=\left|\mathrm{C}_{4}\right\rangle\left\langle\mathrm{C}_{4}\right|$ will prepare various LOCC inequivalent states, like a fourqubit GHZ and Dicke state, as well as into two bipartite maximally entangled states, i.e. a product of Bell states. In general, applying $E_{0}$ given in Eq. (4) on the second and third qubits of $\left|\mathrm{C}_{4}\right\rangle$, we find the general output

$$
\begin{aligned}
E_{0}\left|\mathrm{C}_{4}\right\rangle= & \frac{1}{2}\left[\left(\alpha_{1}-\beta_{1}\right)|H H H H\rangle-\left(\alpha_{2}-\beta_{2}\right)|V V V V\rangle\right. \\
& +\mu_{1}|H H V V\rangle+\mu_{1}|V V H H\rangle \\
& \left.-\mu_{2}|H V H V\rangle-\mu_{2}|V H V H\rangle\right]
\end{aligned}
$$

which by tuning the different coefficients enables the generation of a variety of states as described in the next subsections. In Fig. 5, we show a selection of output states that can be obtained by operating the conversion gate on qubits 2 and 3 of the cluster state together with their success probabilities.

\section{A. Transforming the cluster state into a GHZ state}

In order to obtain a four-qubit GHZ state, $\left|\mathrm{GHZ}_{4}\right\rangle=$ $(|H H H H\rangle+|V V V V\rangle) / \sqrt{2}$, from $\left|\mathrm{C}_{4}\right\rangle$ we should design the evolution operator such that it discards the $|H H V V\rangle$ and $|V V H H\rangle$ components of $\left|\mathrm{C}_{4}\right\rangle$ and flips the minus sign in front of the $|V V V V\rangle$ component. This

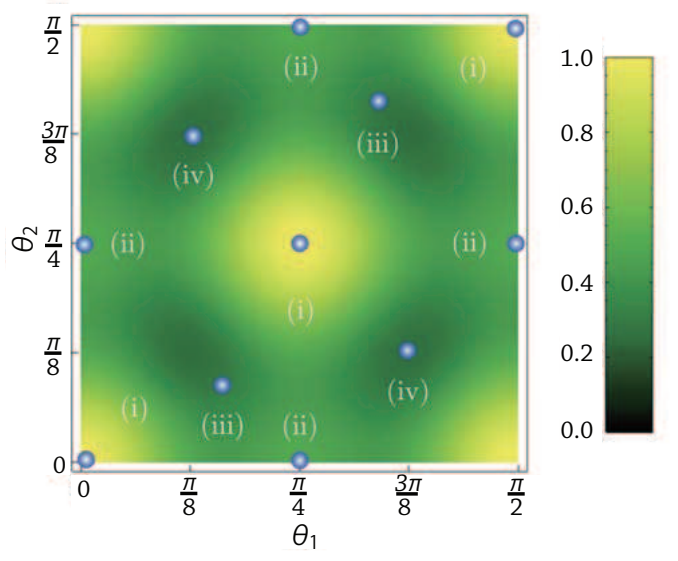

FIG. 5: The success probability for nonlocal state conversion from the linear cluster state $\left|\mathrm{C}_{4}\right\rangle$ to various four-qubit entangled states. See Tab. @for the labelled states and their success probabilities.

can be achieved with an operation element with components $|H H\rangle\langle H H|$ and $|V V\rangle\langle V V|$. Thus, for the output state in Eq. (7) to be $\left|\mathrm{GHZ}_{4}\right\rangle$, we should set $\alpha_{j}$ and $\beta_{j}$ such that $\left(\alpha_{2}-\beta_{2}\right)=-\left(\alpha_{1}-\beta_{1}\right)$ and $\mu_{j}=0$. The latter equality is satisfied for $\left(\theta_{1}, \theta_{2}\right)=\left(m \frac{\pi}{2},(2 n+1) \frac{\pi}{4}\right)$ or $\left(\theta_{1}, \theta_{2}\right)=\left((2 n+1) \frac{\pi}{4}, m \frac{\pi}{2}\right)$ for non-negative integers $m$ and $n$. Substituting these in the former equality leads to $\alpha_{1}-\beta_{1}= \pm 1$ and $\alpha_{2}-\beta_{2}=\mp 1$. Choosing these coefficients yields the Kraus operator of the evolution given by

$$
E_{0}= \pm(|H H\rangle\langle H H|-| V V\rangle\langle V V|)
$$

which achieves the task of transforming $\left|\mathrm{C}_{4}\right\rangle$ to $\left|\mathrm{GHZ}_{4}\right\rangle$ (up to a global phase) with a success probability of $p_{s}=$ $1 / 2$. A Monte Carlo simulation with a $\pm 10 \%$ variation on the wave plate angles gives a success probability of $p_{s}=0.457 \pm 0.027$.

\section{B. Identity operator: Keeping the cluster state intact}

If we do not wish to convert $\left|\mathrm{C}_{4}\right\rangle$ to any other state but keep it intact, while still using the nonlocal gate (for practical reasons re-routing the photons may not be a straightforward procedure), we see from Eq. (7) that in order to obtain $E_{0}=\mathbb{1}$ the coefficients $\mu_{2}=0$ and $\alpha_{1}-\beta_{1}=\alpha_{2}-\beta_{2}=\mu_{1}= \pm 1$ are to be satisfied for $p_{s}=1$. The former equality has the solutions $\left(\theta_{1}, \theta_{2}\right)=\left(m \frac{\pi}{2}, n \frac{\pi}{2}\right)$, with $m$ and $n$ as non-negative integers. We find $\alpha_{j}-\beta_{j}=1$ and $\mu_{1}=(-1)^{n+m}$ from which we obtain $\theta_{j}=k \pi$ and $\theta_{j}=(2 k+1) \frac{\pi}{2}$, respectively, for even and odd $m$. Then the output state of Eq. (7) will be equivalent to $\left|\mathrm{C}_{4}\right\rangle$ if $n+m$ is an even number with a success probability of $p_{s}=1$. With a $\pm 10 \%$ variation on the wave plate angles, $p_{s}=0.909 \pm 0.056$. In this case, the Kraus operator for the successful transformation is 


\begin{tabular}{|r|r|r|r|}
\hline \hline Converted state & $\theta_{1}$ & $\theta_{2}$ & $p_{s}$ \\
\hline (i) Cluster state & 0 & 0 & 1 \\
& $\pi / 2$ & $\pi / 2$ & 1 \\
\hline (ii) GHZ state & $0(\pi / 2)$ & $\pi / 4$ & $1 / 2$ \\
& $\pi / 4$ & $0(\pi / 2)$ & $1 / 2$ \\
\hline (iii) Dicke state & $\theta_{+}$ & $\theta_{-}$ & $3 / 10$ \\
& $\theta_{-}$ & $\theta_{+}$ & $3 / 10$ \\
\hline (iv) Two Bell states & $3 \pi / 8$ & $\pi / 8$ & $1 / 4$ \\
& $\pi / 8$ & $3 \pi / 8$ & $1 / 4$ \\
\hline \hline
\end{tabular}

TABLE I: The success probability for nonlocal state conversion from the linear cluster state $\left|\mathrm{C}_{4}\right\rangle$ to a four-qubit $\mathrm{GHZ}$ and Dicke and two Bell states. The angle $\theta_{ \pm}$is found from the relation, $\sin 2 \theta_{ \pm}=\sqrt{(5 \pm \sqrt{5}) / 10}$.

the desired identity operator

$$
\begin{aligned}
E_{0}= & |H H\rangle\langle H H|+| V V\rangle\langle V V| \\
& +|H V\rangle\langle H V|+| V H\rangle\langle V H| .
\end{aligned}
$$

\section{Transforming the cluster state into two Bell states}

With a proper choice of the coefficients, we can disentangle the four qubit entanglement in the cluster state to prepare two Bell states, one shared between modes 2 and 3 , and the other between the modes 1 and 4 . Note that only the photons in modes 2 and 3 enter the conversion gate. It is easy to see that among all possible settings of the coefficients the setting $\alpha_{1}-\beta_{1}=\alpha_{2}-\beta_{2}=0$ and $\mu_{2}=-\mu_{1}$ leads to the desired transformation. Imposing these conditions results in

$$
\begin{aligned}
E_{0}\left|\mathrm{C}_{4}\right\rangle= & \frac{\mu_{1}}{2}(|H H V V\rangle+|V V H H\rangle \\
& +|H V H V\rangle+|V H V H\rangle) \\
= & \mu_{1}\left|\Psi^{+}\right\rangle_{14}\left|\Psi^{+}\right\rangle_{23} .
\end{aligned}
$$

Thus, if we can find the angles satisfying the above expressions for the coefficients, the desired task will be accomplished with the success probability $p_{s}=\mu_{1}^{2}$. Here we note that the operation required is the same as the one of the first example given in Section II B. Thus, substituting the angles for this example into the expressions given in Section II B gives $\mu_{1}=-\mu_{2}= \pm 1 / 2$. The success probability is $p_{s}=\mu_{1}^{2}=\frac{1}{4}$. With a $\pm 10 \%$ variation on the wave plate angles, $p_{s}=0.270 \pm 0.017$. The Kraus operator that performs this task is

$$
\begin{aligned}
E_{0}= & \frac{1}{2}[|H V\rangle\langle H V|+| V H\rangle\langle H V|+| V H\rangle\langle V H| \\
& +|H V\rangle\langle V H|] .
\end{aligned}
$$

\section{Transforming the cluster state into a Dicke state}

Finally, we show that the nonlocal gate can be used to convert the linear cluster state $\left|\mathrm{C}_{4}\right\rangle$ into the four-qubit Dicke state,

$$
\begin{aligned}
\left|\mathrm{D}_{4}^{(2)}\right\rangle & =\frac{1}{\sqrt{6}}(|H V V H\rangle+|V H H V\rangle+|H V H V\rangle \\
& +|V H V H\rangle+|H H V V\rangle+|V V H H\rangle) .
\end{aligned}
$$

Consider rotating the polarization of the qubits in this state locally via the operation $\sigma_{z} \otimes \sigma_{x} \otimes \sigma_{z} \sigma_{x} \otimes \mathbb{1}$ to give the following state,

$$
\begin{aligned}
\left|\mathrm{D}_{4}^{\prime(2)}\right\rangle & =\frac{1}{\sqrt{6}}(|H H H H\rangle+|V V V V\rangle-|H H V V\rangle \\
& -|V V H H\rangle+|H V H V\rangle+|V H V H\rangle) .
\end{aligned}
$$

It is now clear that if we can set $\beta_{2}-\alpha_{2}=\alpha_{1}-\beta_{1}=$ $-\mu_{1}=\mu_{2}$ in Eq. (77), the coefficients will be equal and the final state will be locally equivalent to a Dicke state. From $\mu_{1}=-\mu_{2}$, we find $\theta_{1}-\theta_{2}=(2 k+1) \frac{\pi}{4}$ which leads to the relations $\sin 2 \theta_{1}=(-1)^{k} \cos 2 \theta_{2}$ and $\cos 2 \theta_{1}=$ $(-1)^{k+1} \sin 2 \theta_{2}$. Using these relations in $\alpha_{2}-\beta_{2}=\mu_{1}$, we obtain $5 \sin ^{4} 2 \theta_{2}-5 \sin ^{2} 2 \theta_{2}+1=0$, whose roots satisfy $\sin ^{2} 2 \theta_{2}=(5 \pm \sqrt{5}) / 10$. Using the expression for $\sin ^{2} 2 \theta_{2}$ in $\sin ^{2} 2 \theta_{1}+\sin ^{2} 2 \theta_{2}=1$, which is derived from the equality $\alpha_{2}-\beta_{2}=\beta_{1}-\alpha_{1}$, we find $\sin ^{2} 2 \theta_{1}=$ $(5 \mp \sqrt{5}) / 10$. Then, setting $\sin ^{2} 2 \theta_{1}=(5 \pm \sqrt{5}) / 10$ gives $\sin ^{2} 2 \theta_{2}=(5 \mp \sqrt{5}) / 10$ and leads to $\alpha_{1}-\beta_{1}=\mp 1 / \sqrt{5}$ and $\alpha_{2}-\beta_{2}=\mu_{1}=\mu_{2}= \pm 1 / \sqrt{5}$. Inserting these values for the coefficients in Eq. (7), we arrive at

$$
\begin{aligned}
& E_{0}\left|\mathrm{C}_{4}\right\rangle= \mp \frac{1}{2 \sqrt{5}}[|H H H H\rangle+|V V V V\rangle-|H H V V\rangle \\
&-|V V H H\rangle+|H V H V\rangle+|V H V H\rangle] \\
&=\mp \frac{\sqrt{6}}{2 \sqrt{5}}\left|\mathrm{D}_{4}^{\prime(2)}\right\rangle
\end{aligned}
$$

implying that we can convert $\left|\mathrm{C}_{4}\right\rangle$ into $\left|\mathrm{D}_{4}^{(2)}\right\rangle$ with a success probability $p_{s}=3 / 10$. The Monte Carlo simulation with a $\pm 10 \%$ variation on the wave plate angles gives a success probability of $p_{s}=0.303 \pm 0.013$. The Kraus operator for the successful transformation is given by

$$
\begin{aligned}
E_{0}= & \mp \frac{1}{\sqrt{5}}(|H H\rangle\langle H H|-| V V\rangle\langle V V|-| H V\rangle\langle H V| \\
& -|V H\rangle\langle V H|+| V H\rangle\langle H V|+| H V\rangle\langle V H|) .
\end{aligned}
$$

\section{NONLOCAL GATE IN A MULTI-QUBIT NETWORK}

We now briefly discuss the integration of the nonlocal gate in a multi-qubit network in the form of a graph state. 
a)

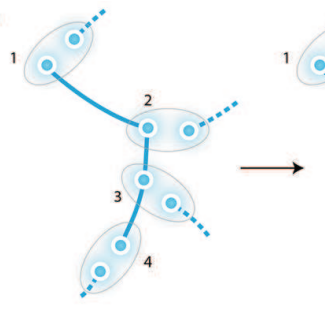

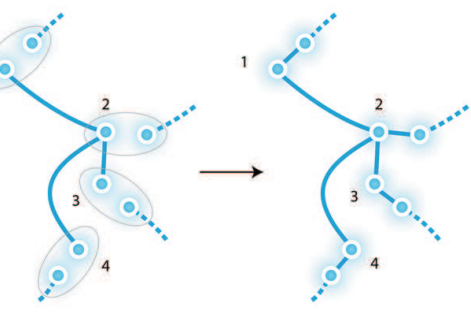

c)

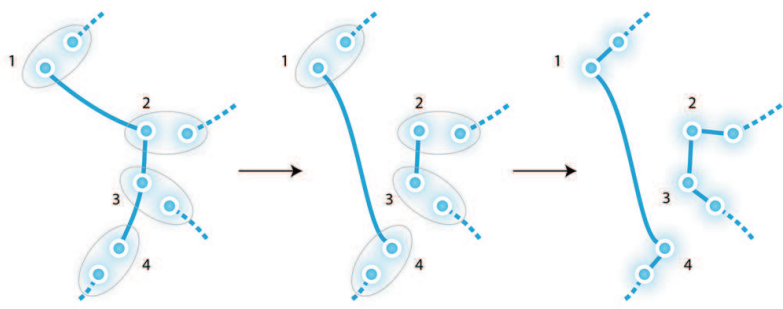

b)

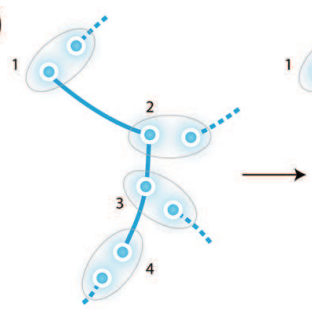

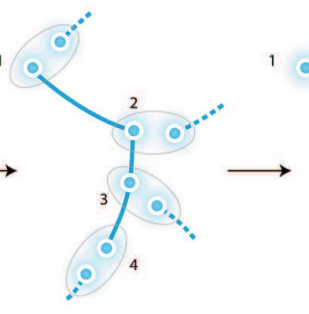

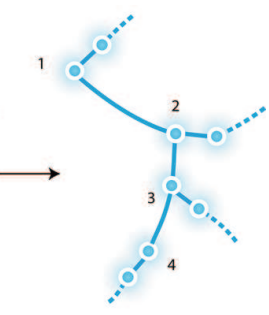

d)

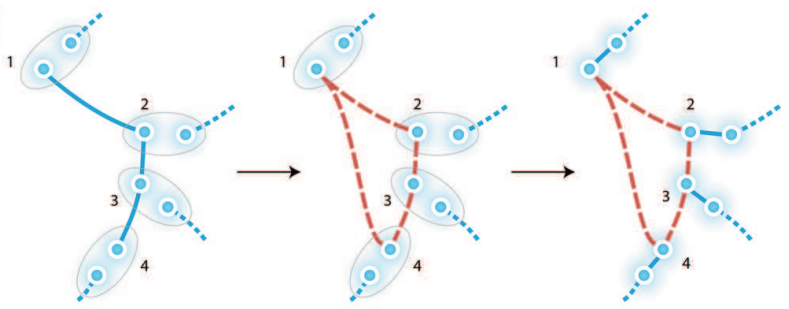

FIG. 6: Nonlocal gate used in a multi-qubit network taking the form of a graph state. Here, vertices are qubits initialized to the state $\frac{1}{\sqrt{2}}(|H\rangle+|V\rangle)$ and edges correspond to the application of a controlled- $Z(C Z)$ operation between the vertices: $C Z=|H\rangle\langle H|\otimes \mathbb{1}+| V\rangle\langle V| \otimes \sigma_{z}$. In the first step of each panel, a linear cluster state has local operations $\mathbf{H} \otimes \mathbb{1} \otimes \mathbb{1} \otimes \mathbf{H}$ applied ( $\mathrm{H}$ is the Hadamard operation) to take it from its canonical graph state form to the state $\left|\mathrm{C}_{4}\right\rangle$. It is then converted into various four-qubit entangled states using the nonlocal gate, as summarized in Tab. I In the second step of each panel, these states are then connected to the rest of the graph representing the network via $C Z$ gates (and local operations). See main text for details. The dashed edges represent $C Z$ gates applied between the qubits shown and other qubits in the total graph (not shown). (a): Cluster state converted into a star graph (locally equivalent to the GHZ state), which is then connected to the rest of the graph of the network. (b): Cluster state remains intact as a cluster state and is connected to the graph (after local operations). (c): Cluster state converted into two 2-qubit graph states (each locally equivalent to a Bell state). (d): Cluster state converted into a Dicke state and connected to the graph to form a hybrid quantum network. Here, the red dashed edges signify the state is entangled and are not $C Z$ operations.

Graph states can be used for a wide variety of quantum networking purposes, including in quantum communication and distributed quantum computation [4, 64]. In this setting an important task is to 'rewire' the network by changing the entanglement structure in order to carry out a specific protocol between selected parties. From the previous section it is clear that the nonlocal gate can convert a cluster state into a number of entangled states with different entanglement structures, as summarized in Tab. I. We now show that as a result the nonlocal gate enables the rewiring of a graph state network.

Consider the initial cluster state as part of a multiqubit graph state, as depicted in the first step of Fig. 6)(a). Here, the cluster state is shown in its canonical graph state form, where vertices correspond to qubits in the state $\frac{1}{\sqrt{2}}(|H\rangle+|V\rangle)$ and solid edges correspond to the application of a controlled- $Z(C Z)$ operation between the vertices: $C Z=|H\rangle\langle H|\otimes \mathbb{1}+| V\rangle\langle V| \otimes \sigma_{z}$. The dashed edges represent $C Z$ gates applied between the qubits shown and other qubits in the total graph state (not shown). The canonical cluster state can be converted into the state $\left|\mathrm{C}_{4}\right\rangle$ with local operations $\mathrm{H} \otimes \mathbb{1} \otimes \mathbb{1} \otimes \mathrm{H}$, where $\mathrm{H}$ is the Hadamard operation. Once these local operations have been performed, the nonlocal gate is then applied. Here, there are four rewiring cases:

(a) Rewiring into a star cluster.- The nonlocal gate is applied between qubits 2 and 3 for the cluster state $\left|\mathrm{C}_{4}\right\rangle$ to become a GHZ state. This state is equivalent to the star cluster state shown in step 2 of Fig. 6 (a) under local operations $\mathbf{H} \otimes \mathbb{1} \otimes \mathbf{H} \otimes \mathbf{H}$. Once these bilocal operations have been performed, $C Z$ operations are then applied to connect the star cluster state into the total graph state, as shown in step 3. This rewires the network. Note that one can choose any qubit to be the central node of the star cluster in step 2 (11 is applied to the central node and $\mathrm{H}$ to the outer nodes), giving four possible rewiring configurations. It is also interesting to note that the star cluster state and linear cluster state are not equivalent under local operations and classical communication [64], making the use of the nonlocal conversion gate necessary in order to rewire the network in this case.

(b) Keeping the initial wiring.- The nonlocal gate is applied between qubits 2 and 3 of the cluster state $\left|\mathrm{C}_{4}\right\rangle$, which remains as $\left|\mathrm{C}_{4}\right\rangle$. This state is equivalent to the linear cluster state shown in step 2 of Fig. 6 (b) under local operations $\mathrm{H} \otimes \mathbb{1} \otimes \mathbb{1} \otimes \mathrm{H}$. Once these local operations have been performed, $C Z$ operations are then applied to connect the linear cluster state into the total graph state. This keeps the initial wiring of the network.

(c) Rewiring into two graphs.- The nonlocal gate is applied between qubits 2 and 3 of the cluster state $\left|\mathrm{C}_{4}\right\rangle$, which becomes a product of two Bell states. Each of these states are equivalent to a two-qubit graph state, as shown in step 2 of Fig. 6 (c), under local operations $\mathbb{1} \otimes \mathrm{H} \sigma_{x}$. Once these local operations have been performed, $C Z$ operations are then applied to connect the graph states into the total graph state. This rewires the network. 
(d) Rewiring into a hybrid network.- The nonlocal gate is applied between qubits 2 and 3, and the corresponding local operations are performed, for the cluster state $\left|\mathrm{C}_{4}\right\rangle$ to become a Dicke state $\left|\mathrm{D}_{4}^{(2)}\right\rangle$. This state is shown in step 2 of Fig. 6 (d), where the internal entanglement connections of the Dicke state (not $C Z$ gates) are represented by dashed red edges. $C Z$ operations are then applied to connect each qubit of the Dicke state individually into the total graph state. This is a standard method for encoding qubits into graph states [4]. The operations produce a hybrid network of a graph state and a Dicke state that may be more efficient for certain distributed protocols, such as in telecloning and quantum secret sharing [44].

\section{CONCLUSION}

In this work we proposed a simple and powerful linear optical quantum gate that can be used to prepare entanglement, or to observe states without entanglement but nonzero discord. In addition, we showed how to convert a four-qubit linear cluster state into a series of relevant multipartite entangled states, such as a four-qubit GHZ, Dicke state and two bipartite maximally entangled states, by acting on only two qubits. The gate can perform a range of nonlocal operations based on its functionality as a tunable polarization dependent beamsplitter. As a re- sult, it can be used to implement various different types of fusion operation in a number of quantum state expansion schemes [23, 24, 32]. Indeed, recent work has also shown how to scale quantum networks by fusing small multipartite entangled states containing four-qubit entanglement 62. Thus, by placing the gate in either a large-scale quantum network [6] or small-scale on-chip network [63], we envisage that it could play an important role in efficiently preparing and converting other types of larger multipartite entangled states where there may be restricted access to a given resource.

\section{Acknowledgments}

This work was supported by a JSPS Postdoctoral Fellowship for Research Abroad. This work was also supported by EU-FET project QWAD, the German excellence research cluster NIM, the South African National Research Foundation and the South African National Institute for Theoretical Physics. TT is supported by Grant-in-Aid for Young Scientists (B), no.15K17729. FN was partially supported by the RIKEN iTHES Project, the MURI Center for Dynamic Magneto-Optics, the IMPACT program of JST, a Grant-in-Aid for Scientific Research (A), and a grant from the John Templeton Foundation.
[1] R. Horodecki et al., "Quantum entanglement", Rev. Mod. Phys. 81, 865 (2009).

[2] N. Gisin et al., "Quantum cryptography", Rev. Mod. Phys. 74, 145 (2002).

[3] H. J. Briegel and R. Raussendorf, "Persistent Entanglement in Arrays of Interacting Particles", Phys. Rev. Lett. 86, 910 (2001); R. Raussendorf and H. J. Briegel, "A One-Way Quantum Computer", Phys. Rev. Lett. 86, 5188 (2001); R. Raussendorf, D. E. Browne and H. J. Briegel, "Measurement-based quantum computation with cluster states", Phys. Rev. A 68, 022312 (2003).

[4] H. J. Briegel et al., "Measurement-based quantum computation", Nat. Phys. 5, 19 (2009).

[5] J.-W. Pan, et al., "Multiphoton entanglement and interferometry", Rev. Mod. Phys. 84, 777 (2012).

[6] H. J. Kimble, "The quantum internet", Nature 453, 1023 (2008).

[7] G. Chiribella, G. M. D'Ariano and P. Perinotti, "Theoretical framework for quantum networks", Phys. Rev. A 80, 022339 (2009).

[8] S. Perseguers, G. J. Lapeyre Jr, D. Cavalcanti, M. Lewenstein and A. Acin, "Distribution of entanglement in largescale quantum networks", Rep. Prog. Phys. 76096001 (2013).

[9] D. M. Greenberger, M. A. Horne, A. Shimony and A. Zeilinger, "Bell's theorem without inequalities", Am. J. Phys. 58, 1131 (1990).

[10] W. Dür, G. Vidal and J. I. Cirac, "Three qubits can be entangled in two inequivalent ways", Phys. Rev. A 62,
062314 (2000).

[11] M. Koashi, V. Bužek and N. Imoto, "Entangled webs: Tight bound for symmetric sharing of entanglement", Phys. Rev. A 62, 050302(R) (2000).

[12] W. Dür, "Multipartite entanglement that is robust against disposal of particles", Phys. Rev. A 63, 020303(R) (2001).

[13] R. H. Dicke, "Coherence in Spontaneous Radiation Processes", Phys. Rev. 93, 99 (1954).

[14] F. Verstraete, J. Dehaene, B. De Moor and H. Verschelde, "Four qubits can be entangled in nine different ways", Phys. Rev. A 65, 052112 (2002).

[15] C. Schmid et al., "Discriminating Multipartite Entangled States", Phys. Rev. Lett. 100, 200407 (2008).

[16] A. Acin, D. Bruss, M. Lewenstein and A. Sanpera, "Classification of Mixed Three-Qubit States", Phys. Rev. Lett. 87, 040401 (2001).

[17] I Buluta, S. Ashhab and F. Nori, "Natural and artificial atoms for quantum computation", Rep. Prog. Phys. 74, 104401 (2011).

[18] L. M. K. Vandersypen and I. L. Chuang, "NMR techniques for quantum control and computation", Rev. Mod. Phys. 76, 1037 (2005).

[19] C. Monroe and J. Kim, "Scaling the Ion Trap Quantum Processor", Science 339, 1164 (2013).

[20] R. Blatt and D. Wineland, "Entangled states of trapped atomic ions", Nature 453, 1008 (2008).

[21] J. Q. You and F. Nori, "Atomic physics and quantum optics using superconducting circuits", Nature 474, 589 
(2011).

[22] Z. -L. Xiang, S. Ashhab, J. Q. You and F. Nori, "Hybrid quantum circuits: Superconducting circuits interacting with other quantum systems", Rev. Mod. Phys. 85, 623 (2013).

[23] D. E. Browne and T. Rudolph, "Resource-Efficient Linear Optical Quantum Computation", Phys. Rev. Lett. 95, 010501 (2005).

[24] T. P. Bodiya and L.-M. Duan, "Scalable Generation of Graph-State Entanglement Through Realistic Linear Optics", Phys. Rev. Lett. 97, 143601 (2006).

[25] N. Kiesel, et al., "Three-photon W-state", J. Mod. Opt. 50, 1131 (2003).

[26] M. Eibl, et al., "Experimental Realization of a ThreeQubit Entangled W State", Phys. Rev. Lett. 92, 077901 (2004).

[27] H. Mikami, Y. Li, K. Fukuoka and T. Kobayashi, "New High-Efficiency Source of a Three-Photon W State and its Full Characterization Using Quantum State Tomography", Phys. Rev. Lett. 95, 150404 (2005).

[28] Y. Li and T. Kobayashi, "Four-photon W state using two-crystal geometry parametric down-conversion", Phys. Rev. A 70, 014301 (2004).

[29] Y. L. Lim and A. Beige, "Multiphoton entanglement through a Bell-multiport beam splitter", Phys. Rev. A 71, 062311 (2005).

[30] K. J. Resch, P. Walther and A. Zeilinger, "Full Characterization of a Three-Photon Greenberger-HorneZeilinger State Using Quantum State Tomography", Phys. Rev. Lett. 94, 070402 (2005).

[31] T. Tashima et al., "Elementary optical gate for expanding an entanglement web", Phys. Rev. A 77, 030302(R) (2008).

[32] T. Tashima et al., "Local expansion of photonic W state using a polarization-dependent beamsplitter", New J. Phys 11, 023024 (2009).

[33] Y- X. Gong, X- B. Zou, Y- F. Huang and G- C. Guo, "A simple scheme for expanding a polarization-entangled $\mathrm{W}$ state by adding one photon", J. Phys. B: At. Mol. Opt. Phys. 42, 035503 (2009).

[34] T. Tashima et al., "Local Transformation of Two Einstein-Podolsky-Rosen Photon Pairs into a ThreePhoton W State", Phys. Rev. Lett. 102, 130502 (2009).

[35] T. Tashima et al., "Demonstration of Local Expansion Toward Large-Scale Entangled Webs", Phys. Rev. Lett. 105, 210503 (2010).

[36] N. Kiesel et al., "Experimental Analysis of a Four-Qubit Photon Cluster State", Phys. Rev. Lett. 95, 210502 (2005).

[37] A. S. Clark et al., "All-optical-fiber polarization-based quantum logic gate", Phys. Rev. A 79, 030303R (2009).

[38] X.-S. Ma et al., "A high-speed tunable beam splitter for feed-forward photonic quantum information processing", Opt. Exp. 19, 22723 (2011).

[39] R. Ikuta et al., "Optimal local expansion of W states using linear optics and Fock states", Phys. Rev. A 83, 012314 (2011).

[40] H. Nha and J. Kim, "Demonstrating multipartite entanglement of single-particle W states: Linear optical schemes", Phys. Rev. A 75, 012326 (2007).

[41] W.-B. Gao et al., "Experimental Realization of a Controlled-NOT Gate with Four-Photon Six-Qubit Cluster States", Phys. Rev. Lett. 104, 020501 (2010).

[42] W. Wieczorek et al., "Experimental Observation of an
Entire Family of Four-Photon Entangled States", Phys. Rev. Lett. 101, 010503 (2008).

[43] W. Wieczorek et al., "Multiqubit entanglement engineering via projective measurements", Phys. Rev. A 79, 022311 (2009).

[44] R. Prevedel et al., "Experimental Realization of Dicke States of up to Six Qubits for Multiparty Quantum Networking", Phys. Rev. Lett. 103, 020503 (2009); W. Wieczorek et al., "Experimental Entanglement of a SixPhoton Symmetric Dicke State", Phys. Rev. Lett. 103, 020504 (2009).

[45] S. Bugu, C. Yesilyurt and F. Ozaydin, "Enhancing the W-state quantum-network-fusion process with a single Fredkin gate", Phys. Rev. A 87, 032331 (2013).

[46] F. Ozaydin et al., "Fusing multiple W states simultaneously with a Fredkin gate", Phys. Rev. A 89, 042311 (2014).

[47] E. D'Hondt and P. Panangaden, "The Computational Power of the W and GHZ states", Quantum Inf. Comput. 6, 173 (2006).

[48] V. Scarani et al., "Quantum cloning", Rev. Mod. Phys. 77, 1225 (2005).

[49] A. Soeda, Y. Kinjo, P. S. Turner and M. Murao, "Quantum computation over the butterfly network", Phys. Rev. A 84, 012333 (2011).

[50] N. Kiesel, et al., "Experimental Observation of FourPhoton Entangled Dicke State with High Fidelity", Phys. Rev. Lett. 98, 063604 (2007).

[51] J. Shimamura et al., "Entangled states that cannot reproduce original classical games in their quantum version", Phys. Lett. A 328, 20 (2004).

[52] S. K. Özdemir, J. Shimamura and N. Imoto "A necessary and sufficient condition to play games in quantum mechanical settings", New J. Phys 9, 43 (2007).

[53] P. Walther, K. J. Resch and A. Zeilinger, "Local Conversion of Greenberger-Horne-Zeilinger States to Approximate W States", Phys. Rev. Lett. 94, 240501 (2005).

[54] W. K. Wootters, "Entanglement of Formation of an Arbitrary State of Two Qubits", Phys. Rev. Lett. 80, 2245 (1998).

[55] L. Henderson and V. Vedral, "Classical, quantum and total correlations", J. Phys. A: Math. Gen. 34, 6899 (2001).

[56] H. Ollivier and W. H. Zurek, "Quantum Discord: A Measure of the Quantumness of Correlations", Phys. Rev. Lett. 88, 017901 (2001).

[57] K. Modi et al., "The classical-quantum boundary for correlations: Discord and related measures", Rev. Mod. Phys. 84, 1655 (2012).

[58] B. Dakić et al., "Quantum discord as resource for remote state preparation", Nat. Phys. 8, 666 (2012).

[59] M. Gu et al., "Observing the operational significance of discord consumption", Nat. Phys. 8, 671 (2012).

[60] M. Almeida et al., "Entanglement-free certification of entangling gates", Phys. Rev. A 89, 042323 (2014).

[61] S. Pirandola, "Quantum discord as a resource for quantum cryptography", Sci. Rep. 4, 6956 (2014).

[62] K. Fujii et al., "A distributed architecture for scalable quantum computation with realistically noisy devices", arXiv:1202.6588v1 (2012).

[63] J. Silverstone et al., "On-chip quantum interference between silicon photon-pair sources", Nature Photon. 8104 (2014).

[64] M. Hein et al., in Proc. Inter. Sch. Phys. "Enrico Fermi" on "Quantum Computers, Algorithms and Chaos", 
Varenna, Italy, July, (2005). 